\title{
BÀN VỀ CHUYỀN ĐỘ CAO GEOID THÀNH ĐỘ CAO QUASIGEOID
}

\author{
HÀ MINH HÒA \\ Viện Khoa học Đo đạc và Bản đồ
}

\section{Tóm tắt:}

Việc nghiên cúu chuyển đổi độ cao geoid thành độ cao quasigeoid là một hướng nghiên cúu của trắc địa vật lý. Bài báo này đã trình bày các nghiên cứu lý thuyết về huớng này và trình bày một số thự nghiệm trên thế giới. Các kết quả nghiên cưu thực nghiệm cho thấy độ chênh giữa độ cao geoid và độ cao quasigeoid là khá lớn và hiện nay không đáp ứng được các yêu cầu hiện đại của việc xây dụng các mô hình geoid/quasigeoid. Điều này nói lên rằng sụ phân bố vật chất giữa mặt vật lý trái đất và mặt geoid rất phức tạp, hiện nay chưa mô hình hóa được.

\section{1. Đặt vấn đề}

Dựa theo lý thuyết Molodensky M.S., mô hình quasigeoid toàn cầu được xây dựng là mô hình xấp xỉ lần thứ nhất của Trái đất. Mô hình thực của Trái đất là mô hình geoid toàn cầu sẽ được tính toán trong những lần xấp xỉ tiếp theo. Theo phương hướng này, trong tài liệu (Heiskanen W.A. and Moritz H., 1967) đã đưa ra công thức chuyển độ cao quasigeoid $\bar{\zeta}$ về độ cao geoid toàn cầu $\bar{N}$ tại điểm P trên mặt vật lý Trái đất theo qua công thức sau:

$$
\bar{\zeta}-\bar{N}=-\frac{\bar{g}-\bar{\gamma}}{\bar{\gamma}} \cdot \bar{H}^{g} \approx-\frac{\Delta g_{P}^{B}}{\bar{\gamma}} \bar{H}^{g},
$$

ở đây $\bar{g}$ - giá trị trung bình tích phân của gia tốc lực trọng trường tương ứng với điểm $\mathrm{P} ; \bar{\gamma}$ - giá trị trung bình của gia tốc lực trọng trường chuẩn tại điểm $\mathrm{P} ; \bar{H}^{g}$ và $\bar{H}^{\gamma}$ - các độ cao chính toàn cầu và độ cao chuẩn toàn cầu của điểm $\mathrm{P}$, thêm vào đó các đại lượng $\bar{H}^{g}$ và $\bar{H}^{\gamma}$ quan hệ với nhau bởi biểu thức:

$$
\bar{H}^{\gamma}-\bar{H}^{g}=\frac{\bar{g}-\bar{\gamma}}{\bar{\gamma}} \cdot \bar{H}^{g} \approx \frac{\Delta g_{P}^{B}}{\bar{\gamma}} \cdot \bar{H}^{g} .
$$

Các công thức (1), (2) hình thành nên một hướng nghiên cứu trong Trắc địa vật lý: Lý thuyết chuyển độ cao quasigeoid $\bar{\zeta}$ về độ cao geoid toàn cầu $\bar{N}$ tại điểm $\mathrm{P}$ trên mặt vật lý Trái đất. Tuy nhiên các công thức trên con chưa tính đến sự hấp dẫn của khối lượng vật chất địa hình giữa mặt vật lý Trái đất và mặt geoid toàn cầu đã tạo ra thế địa hình (topographic potential) $V_{g}^{T}$ trên mặt geoid và thế địa hình $V_{P}^{T}$ tại điểm trọng lực $\mathrm{P}$ trên mặt vật lý Trái đất.

Trong bài báo khoa học này, chúng ta sẽ xem xét việc phát triển của công thức (1) và một số thử nghiệm chuyển độ cao quasigeoid $\bar{\zeta}$ về độ cao geoid toàn cầu $\bar{N}$ tại điểm $\mathrm{P}$ trên mặt vật lý Trái đất.

\section{Các kết quả nghiên cứu}

Trong chương 6 của tài liệu Hà Minh Hòa, 2018 đã phân tích khá chi tiết các kết quả nghiên cứu chuyển độ cao quasigeoid $\bar{\zeta}$ về độ cao geoid toàn cầu $\bar{N}$ tại điểm P trên mặt vật lý Trái đất. Trong quá trình hoàn tiện các công thức (1), (2), bên cạnh việc tính đến sự hấp dẫn của khối lượng vật chất 
địa hình giữa mặt vật lý Trái đất và mặt geoid toàn cầu đã tạo ra thế địa hình (topographic potential) $V_{g}^{T}$ trên mặt geoid và thế địa hình $V_{P}^{T}$ tại điểm trọng lực $\mathrm{P}$ trên mặt vật lý Trái đất. Ngoài ra còn phải tính đến dị thường Bouguer $\Delta g_{P}^{B}$ tại điểm $\mathrm{P}$ là độ nhiễu trọng lực (gravity disturbance) $\delta g^{B}$ trên mặt geoid toàn cầu. Do sự thay đổi của độ nhiễu trọng lực $\delta g^{B}$ từ điểm $\mathrm{P}$ đến mặt biển, nên trong tài liệu (Flury J., Rummel R., 2009) đã đề xuất công thức chặt chẽ để chuyển dị thường độ cao toàn cầu thành độ cao geoid toàn cầu ở dạng sau:

$$
\bar{N}-\bar{\zeta}=\frac{\Delta g_{P}^{B}}{\bar{\gamma}} \cdot \bar{H}^{g}+T C 1+G C 1,
$$

ở đây số hiệu chỉnh địa hình TC1 (topographic correction) có dạng:

$$
T C \mathrm{l}=\frac{V_{g}^{T}-V_{P}^{T}}{\bar{\gamma}},
$$

còn số hiệu chỉnh trọng lực GC1 (gravimetric correction) có dạng:

$$
G C 1=\frac{\delta \bar{g}^{B}-\delta g_{P}^{B}}{\bar{\gamma}},
$$

$\delta g_{P}^{B}$ - độ nhiễu trọng lực tại điểm $\mathrm{P} ; \delta \bar{g}^{B}$ - độ nhiễu trọng lực trung bình dọc theo độ cao từ điểm $\mathrm{P}$ đẹn mặt biển; $\bar{\gamma}$ - giá trị trung bình của gia tốc lực trọng trường chuẩn tại điểm $\mathrm{P}$.

Trong tài liệu (Sjöberg Lars E., 2010) đã hoàn thiện tiếp theo công thức (3) ở dạng sau:

$$
\bar{N}_{P}-\bar{\zeta}_{P}=\frac{\Delta g_{P}^{B}}{\bar{\gamma}} \bar{H}^{g}+T C+G C
$$

ở đây số hiệu chỉnh địa hình TC (Topographic Correction) có dạng:

$$
T C=\frac{V_{g}^{T}}{\gamma_{0}}-\frac{V_{P}^{T}}{\gamma_{N}}-\frac{V_{P}^{T}}{\bar{\gamma} \cdot \gamma_{N}} \cdot \frac{\partial \gamma}{\partial h} \cdot \bar{H}_{P}^{g},
$$

còn số hiệu chỉnh trọng lực GC (Gravity Correction) có dạng:

$$
G C=\int_{0}^{\bar{H}_{P}^{g}}\left(\frac{\Delta g^{N T}}{\gamma}-\frac{\Delta g_{P}^{N T}}{\gamma_{N}}\right) \cdot d h,
$$

$\Delta g^{N T}$ - dị thường trọng lực không bị ảnh hưởng của địa hình theo độ cao từ điểm $\mathrm{P}$ đến mặt biển; $\Delta g_{P}^{N T}$ - dị thường trọng lực không bị ảnh hưởng của địa hình tại điểm $\mathrm{P}$.

Trong tài liệu (Sjöberg Lars E., 2010) không chứng minh các công thức (6), (7), (8) mà chỉ so sánh chúng với các công thức (3), (4), (5). Trong phần tiếp theo, chúng ta sẽ nghiên cứu cơ sở lý luận của các công thức $(6),(7),(8)$. Trước tiên, chúng ta thừa nhận rằng về mặt lý thuyết, dị thường Bouguer $\Delta g_{P}^{B}$ đã được loại bỏ ảnh hưởng của địa hình giữa điểm $\mathrm{P}$ trên mặt vật lý Trái đất và mặt geoid toàn cầu và đã được đưa xuống mặt geoid này. Do đó các giá trị dị thường độ cao toàn cầu $\bar{\zeta}$ và độ cao geoid toàn cầu $\bar{N}$ trong công thức (1) là các giá trị không bị ảnh hưởng của địa hình (No - Topography - NT), tức $\bar{\zeta}=\bar{\zeta}^{N T}, \bar{N}=\bar{N}^{N T}$ Mặt khác, độ cao geoid $\bar{N}^{N T}$ lại được tính toán trên mặt geoid toàn cầu, còn dị thường độ cao $\bar{\zeta}^{N T}=\bar{\zeta}_{P}^{N T}$ lại được tính toán tại điểm $\mathrm{P}$ trên mặt vật lý Trái đất. Như chúng ta sẽ thấy ở phần dưới đây, sự thay đổi của độ cao geoid toàn cầu $\bar{N}_{P}^{N T}$ theo độ 
cao được xác định theo công thức

$$
\frac{\partial \bar{N}_{P}^{N T}}{\partial h}=-\frac{\Delta g_{P}^{B}}{\gamma}
$$

Do đó từ (1) suy ra $\bar{\zeta}_{P}^{N T}=\bar{N}^{N T}+\frac{\partial \bar{N}_{P}^{N T}}{\partial h} \bar{H}_{P}^{g}$. Công thức này có nghĩa là độ cao geoid toàn cầu $\bar{N}^{N T}$ trên mặt geoid toàn cầu được thành dị thường độ cao toàn cầu $\bar{\zeta}_{P}^{N T}$ ở mức của điểm $\mathrm{P}$. Khi đó chúng ta viết lại công thức (1) ở dạng sau:

$$
\left(\bar{N}^{N T}-\bar{\zeta}^{N T}\right)_{P}=\frac{\Delta g_{P}^{B}}{\bar{\gamma}} \bar{H}_{P}^{g}
$$

Công thức (9) chỉ ra rằng hiệu $\left(\bar{N}^{N T}-\bar{\zeta}^{N T}\right)_{P}$ được xác định trên mức của điểm $\mathrm{P}$. Trong thực tế, độ cao geoid toàn cầu $\bar{N}$ được xác định trên mặt geoid toàn cầu và là độ cao của điểm $\mathrm{Q}$ trên mặt geoid toàn cầu so với mặt ellipsoid Trái đất trung bình, thêm vào đó điểm $\mathrm{Q}$ nằm trên đường dây dọi từ điểm $\mathrm{P}$ đến mặt geoid toàn cầu. Nếu coi dị thường độ cao toàn cầu $\bar{\zeta}$ được xác định trên mặt quasigeoid toàn cầu và là độ cao của điểm $\mathrm{Q}_{0}$ trên mặt quasigeoid toàn câu so với mặt ellipsoid Trái đất trung bình, thêm vào đó điểm $\mathrm{Q}_{0}$ nằm trên đường dây vuông góc với mặt ellipsoid này từ điểm $\mathrm{P}$ đến mặt quasigeoid toàn cầu. Khi đó, chúng ta cần xác định hiệu $(\bar{N}-\bar{\zeta})_{g}$ là độ chênh của mặt quasigeoid toàn cầu so với mặt geoid toàn cầu tại điểm $Q$ trên mặt geoid toàn cầu. Ký hiệu "g" là chữ cái đầu của từ "geoid".

Chúng ta sẽ làm rõ một số đại lượng vật lý không phụ thuộc địa hình. Về mặt lý thuyết, độ cao geoid toàn cầu được xác định theo công thức (Sjöberg Lars E., 2010):

$$
\bar{N}_{P}=\frac{T_{g}}{\gamma_{0}},
$$

ở đây $T_{g}$ là thế nhiễu tại điểm $\mathrm{Q}$ trên mặt geoid toàn cầu, $\gamma_{0}$ - gia tốc lực trọng trường chuẩn tại điểm tương ứng với điểm $\mathrm{P}$ trên mặt ellipsoid Trái đất trung bình theo đường vuông góc từ điểm $\mathrm{P}$ đến mặt ellipsoid này, còn dị thường độ cao toàn cầu được xác định theo công thức:

$$
\bar{\zeta}_{P}=\frac{T_{P}}{\gamma_{N}},
$$

ở đây $T_{P}$ là thế nhiễu tại điểm $\mathrm{P}$ trên mặt vật lý Trái đất, $\gamma_{\mathrm{N}}$ - là gia tốc lực trọng trường chuẩn tại điểm $\mathrm{N}$ tương ứng với điểm $\mathrm{P}$ trên mặt telluroid.

Nếu loại bỏ thế địa hình $V_{g}^{T}$ trong thế nhiễu $T_{g}$ chúng ta nhận được thế nhiễu không bị ảnh hưởng của địa hình $T_{g}^{N T}=T_{g}-V_{g}^{T}$. Khi đó, từ (10) chúng ta có công thức xác định độ cao geoid toàn cầu không bị ảnh hướng cúa địa hình ở dạng sau:

$$
\bar{N}_{P}^{N T}=\frac{T_{g}-V_{g}^{T}}{\gamma_{0}}=\frac{T_{g}^{N T}}{\gamma_{0}}=\frac{T_{g}}{\gamma_{0}}-\frac{V_{g}^{T}}{\gamma_{0}}=\bar{N}_{P}-\frac{V_{g}^{T}}{\gamma_{0}} .
$$

Tương tự, nếu loại bỏ thế địa hình $V_{P}^{T}$ trong thế nhiễu $T_{P}$ chúng ta nhận được thế nhiễu không bị ảnh hưởng của địa hình $T_{P}^{N T}=T_{P}-V_{P}^{T}$. Khi đó, từ (11) chúng ta có công thức xác định dị thường độ cao toàn cầu không bị ảnh hưởng của địa hình ở dạng sau:

$$
\bar{\zeta}_{P}^{N T}=\frac{T_{P}-V_{P}^{T}}{\gamma_{N}}=\frac{T_{P}^{N T}}{\gamma_{N}}=\frac{T_{P}}{\gamma_{N}}-\frac{V_{P}^{T}}{\gamma_{N}}=\bar{\zeta}_{P}-\frac{V_{P}^{T}}{\gamma_{N}} .
$$


Với mục đích xác định $(\bar{N}-\bar{\zeta})_{g}$ tại điểm Q trên mặt geoid toàn cầu, từ (12) và (13) chúng ta có:

$$
(\bar{N}-\bar{\zeta})_{g}=\left(\bar{N}^{N T}-\bar{\zeta}^{N T}\right)_{g}+\left(\frac{V_{g}^{T}}{\gamma_{0}}-\frac{V_{P}^{T}}{\gamma_{N}}\right)
$$

Chúng ta sẽ xác định đại lượng $\left(\bar{N}^{N T}-\bar{\zeta}^{N T}\right)_{g}$ trong công thức (14). Do trong dị thường Bouguer $\Delta g_{P}^{B}$ đã loại bỏ ảnh hưởng của khối lượng vật chất địa hình, nên từ (12) chúng ta có:

$$
\frac{\partial \bar{N}_{P}^{N T}}{\partial h}=\frac{\partial}{\partial h}\left(\frac{T_{g}^{N T}}{\gamma_{0}}\right)=\frac{\partial}{\partial \gamma} \cdot\left(\frac{T_{g}^{N T}}{h}\right)=-\frac{\Delta g_{P}^{B}}{\gamma} .
$$

Tương tự, từ (13) suy ra $\frac{\partial \bar{\zeta}_{P}^{N T}}{\partial h}=\frac{\partial}{\partial h}\left(\frac{T_{P}^{N T}}{\gamma_{N}}\right)=\frac{\partial}{\partial \gamma} \cdot\left(\frac{T_{P}^{N T}}{h}\right)=-\frac{\Delta g_{P}^{N T}}{\gamma_{N}}$.

Lấy hiệu hai biển thức trên, chúng ta nhận được:

$$
\frac{\partial\left(\bar{N}^{N T}-\bar{\zeta}^{N T}\right)}{\partial h}=-\frac{\Delta g_{P}^{B}}{\gamma}+\frac{\Delta g_{P}^{N T}}{\gamma_{N}} .
$$

Mặt khác, giữa dị thường Bouguer và dị thương không khí tự do tồn tại quan hệ (Sjöberg Lars E., 2010):

$$
\Delta g^{B}=\Delta g^{N T}-\frac{V_{P}^{T}}{\gamma_{N}} \cdot \frac{\partial \gamma}{\partial h} .
$$

Khi đó, công thức (16) có dạng mới:

$$
\frac{\partial\left(\bar{N}^{N T}-\bar{\zeta}^{N T}\right)}{\partial h}=-\left(\frac{\Delta g^{N T}}{\gamma}-\frac{\Delta g_{P}^{N T}}{\gamma_{N}}\right)+\frac{V_{P}^{T}}{\gamma \cdot \gamma_{N}} \cdot \frac{\partial \gamma}{\partial h} .
$$

Bây giờ lấy tích phân hai về của công thức (17) với độ cao h thay đổi từ 0 đến độ cao chính toàn cầu $\bar{H}_{P}^{g}$ của điểm $\mathrm{P}$, chúng ta nhận được:

$$
\begin{aligned}
& \int_{0}^{\bar{H}_{P}^{g}} \frac{\partial\left(\bar{N}^{N T}-\bar{\zeta}^{N T}\right)}{\partial h} \cdot d h=\left(\bar{N}^{N T}-\bar{\zeta}^{N T}\right)_{P}-\left(\bar{N}^{N T}-\bar{\zeta}^{N T}\right)_{g}= \\
& =-\int_{0}^{\bar{H}_{P}^{g}}\left(\frac{\Delta g^{N T}}{\gamma}-\frac{\Delta g_{P}^{N T}}{\gamma_{N}}\right) \cdot d h+\int_{0}^{\bar{H}_{P}^{g}} \frac{V_{P}^{T}}{\gamma_{N}} \cdot \frac{\partial \gamma}{\partial h} \cdot d h= \\
& =-G C+\frac{V_{P}^{T} \cdot \bar{H}_{P}^{g}}{\bar{\gamma} \cdot \gamma_{N}} \cdot \frac{\partial \gamma}{\partial h},
\end{aligned}
$$

ở đây GC có dạng (8).

Lưu ý (9), từ (18) chúng ta có: $\left(\bar{N}^{N T}-\bar{\zeta}^{N T}\right)_{g}=\frac{\Delta g_{P}^{B}}{\bar{\gamma}} \bar{H}_{P}^{g}+G C-\frac{V_{P}^{T} \cdot \bar{H}_{P}^{g}}{\bar{\gamma} \cdot \gamma_{N}} \cdot \frac{\partial \gamma}{\partial h}$.

Chúng ta sẽ phân tích số hiệu chỉnh GC (8). Lưu ý (15), công thức (8) được biến đổi như sau: 


$$
\begin{aligned}
& G C=\int_{0}^{\bar{H}_{P}^{g}}\left(\frac{\Delta g^{N T}}{\gamma}-\frac{\Delta g_{P}^{N T}}{\gamma_{N}}\right) \cdot d h=-\int_{0}^{\bar{H}_{P}^{g}}\left(\frac{\delta \bar{\zeta}^{N T}}{\partial h}+\frac{\Delta g_{P}^{N T}}{\gamma_{N}}\right)= \\
& =\bar{\xi}_{g}^{N T}-\zeta_{P}^{N T}-\frac{\Delta g_{P}^{N T}}{\gamma_{N}} \cdot \bar{H}_{P}^{g} .
\end{aligned}
$$

Như vậy, công thức (19) biểu thị sự thay đổi của dị thường độ cao toàn cầu không phụ thuộc địa hình (trong không khí tự do) từ mặt vật lý Trái đất đến mặt geoid toàn cầu. Trong tài liệu (Lars E. Sjöberg, 2010) đã biểu diễn công thức (19) dưới dạng khai triển chuỗi Taylor ở dạng sau:

$$
G C=\sum_{k=2}^{\infty} \frac{\left(\bar{H}_{P}^{g}\right)^{k}}{k ! \cdot \gamma_{N}} \cdot(-1)^{k} \cdot\left(\frac{\delta \bar{\xi}^{N T}}{\partial h}\right)_{P}^{k} \approx-\frac{\left(\bar{H}_{P}^{g}\right)^{2}}{2 \cdot \gamma_{N}} \cdot\left(\frac{\partial \Delta g_{P}^{N T}}{\partial h}\right)_{P} .
$$

Từ công thức (1.114) trong Hà Minh Hòa, 2018, chúng ta viết lại công thức trên dưới dạng:

$$
G C=-\frac{R^{2} \cdot\left(\bar{H}_{P}^{g}\right)^{2}}{4 \pi \cdot \gamma_{N}} \cdot \int_{\Sigma} \frac{\left(\Delta g-\Delta g_{P}\right)}{l_{0}^{3}} \cdot d \Sigma^{\prime} .
$$

Chúng ta sẽ phân tích số hiệu chỉnh GC (8). Lưu ý (15), công thức (8) được biến đổi như sau:

$$
\begin{aligned}
& G C=\int_{0}^{\bar{H}_{P}^{g}}\left(\frac{\Delta g^{N T}}{\gamma}-\frac{\Delta g_{P}^{N T}}{\gamma_{N}}\right) \cdot d h=-\int_{0}^{\bar{H}_{P}^{g}}\left(\frac{\delta \bar{\zeta}^{N T}}{\partial h}+\frac{\Delta g_{P}^{N T}}{\gamma_{N}}\right)= \\
& =\bar{\zeta}_{g}^{N T}-\zeta_{P}^{N T}-\frac{\Delta g_{P}^{N T}}{\gamma_{N}} \cdot \bar{H}_{P}^{g} .
\end{aligned}
$$

Như vậy, công thức (21) biểu thị sự thay đổi của dị thường độ cao toàn cầu không phụ thuộc địa hình (trong không khí tự do) từ mặt vật lý Trái đất đến mặt geoid toàn cầu. Trong tài liệu (Lars E. Sjöberg, 2010) đã biểu diễn công thức (21) dưới dạng khai triển chuỗi Taylor ở dạng sau:

$$
G C=\sum_{k=2}^{\infty} \frac{\left(\bar{H}_{P}^{g}\right)^{k}}{k ! \cdot \gamma_{N}} \cdot(-1)^{k} \cdot\left(\frac{\delta \bar{\zeta}^{N T}}{\partial h}\right)_{P}^{k} \approx-\frac{\left(\bar{H}_{P}^{g}\right)^{2}}{2 \cdot \gamma_{N}} \cdot\left(\frac{\partial \Delta g_{P}^{N T}}{\partial h}\right)_{P} .
$$

Từ công thức (1.114) trong Hà Minh Hòa, 2018, chúng ta viết lại công thức trên dưới dạng:

$$
G C=-\frac{R^{2} \cdot\left(\bar{H}_{P}^{g}\right)^{2}}{4 \pi \cdot \gamma_{N}} \cdot \int_{\Sigma}^{\left(\Delta g-\Delta g_{P}\right)} \frac{\left(d \Sigma^{\prime}\right.}{l_{0}^{3}} .
$$

Khi coi vùng tích phân $d \omega^{\prime}=d \Sigma$.dh, công thức (1.131) trong Hà Minh Hòa, 2018 có dạng:

$$
g_{1}=-\frac{R^{2}}{2 \pi} \cdot \int_{\Sigma} \int_{0}^{\bar{H}_{P}^{g}} \frac{\bar{H}^{g}-\bar{H}_{P}^{g}}{l_{0}^{3}} \cdot\left(\Delta g-\Delta g_{P}\right) \cdot d \Sigma \cdot d h=\frac{R^{2} \cdot\left(\bar{H}_{P}^{g}\right)^{2}}{4 \pi} \cdot \int_{\Sigma} \frac{\left(\Delta g-\Delta g_{P}\right)}{l_{0}^{3}} \cdot d \Sigma \text {. }
$$

So sánh (22) và (23) chúng ta nhận được quan hệ $G C=-\frac{g_{1}}{\gamma_{N}}$.

Từ (19) lưu ý (15) chúng ta có quan hệ:

$$
\bar{\zeta}_{g}^{N T}=\bar{\zeta}_{P}^{N T}+\frac{\partial \bar{\zeta}_{P}^{N T}}{\partial h} \cdot \bar{H}_{P}^{g}+G C
$$


Thành phần thứ hai ở vế phải của (24) là số hiệu chỉnh được sử dụng để chuyển dị thường độ cao toàn cầu $\bar{\zeta}_{P}^{N T}$ từ điểm $\mathrm{P}$ trên mặt vật lý Trái đất về mặt geoid toàn cầu, còn số hiệu chỉnh GC được sử dụng để hiệu chỉnh dị thường độ cao toàn cầu $\bar{\zeta}_{P}^{N T}+\frac{\partial \bar{\zeta}_{P}^{N T}}{\partial h} \cdot H_{P}^{g}$ do sự không song song của mặt đẳng thế đi qua điểm $\mathrm{P}$ so với mặt geoid toàn cầu.

\section{Các kết quả thực nghiệm trên thế giới}

Theo tài liệu Foroughi, I., Tenzer,R., 2017, khi tính toán ở vùng Himalaya và Tibet, độ chênh giữa độ cao quasigeoid và độ cao geoid cực đại đạt mức -4,06 m. Theo tài liệu Dawod,G., Mohamed, H.F., 2009, khi sử dụng mô hình EIGEN-CG01C, độ chênh này đạt mức 0,46-0,52 m. Theo tài liệu Flury, J., Rummel, R., 2009, ở các vùng có đặc trưng địa hình phức tạp, độ chênh này có thể đạt tới $3 \mathrm{~m}$. Theo tài liệu Sjöberg, Lars E., 2018, độ chênh này có thể đạt mức $5 \mathrm{~m}$.

Các kết quả nghiên cứu cho thấy hiện nay việc chuyển đổi độ cao geoid thành độ cao geoid chưa cho kết quả mong muốn với độ chính xác cao. Điều này nói lên rằng sự phân bố vật chất giữa mặt vật lý trái đất và mặt geoid rất phức tạp, chưa mô hình hóa được.

Nhiều phần mềm tính toán độ cao geoid được cấp trên mạng, ví dụ GRAVSOFT, có công cụ chuyển độ cao geoid thành độ cao quasigeoid (Tscherning, C., Forsberg, RE., Knudsen, P., 1992). Khi sử dụng phần mềm này để xử lý tính toán độ cao geoid và sử dụng công cụ chuyển độ cao geoid thành độ cao quasigeoid ở Việt Nam cần hết sức thận trọng do sự chưa chính xác của việc chuyển độ cao geoid thành độ cao quasigeoid./.O

\section{Tài liệu tham khảo}

[1]. Dawod,G., Mohamed, H.F., 2009. Fitting Gravimetric Local and Global Quasi-geoid to GPS/Lavelling Data: The role of Geoid/Quasi-Geoid Variantoins. JKAU: Eng. Sci., Vol.20 No.1, pp: 47-59 (2009 A.D. / 1430 A.H.), DOI: 10.4197/Eng.20-1.3

[2]. Foroughi, I., Tenzer,R., 2017. Comparision of different methods for estimating the geoid-toquasi-geoid separation. Geophysical Journal International, Volume 210, Issue 2,pp.1001-1020, https://doi.org/10.1093/gji/ggx221

[3]. Hà Minh Hòa, 2018. Một số vấn đề hiện đại của Trắc địa vật lý. 964 trg.(Sách chuyên khảo). ISBN: 978- 604-67-1116-2, Nhà Xuất bản Khoa học và Kỹ thuật.

[4]. Heiskanen, W.A., and Moritz, H., 1967. Physical Geodesy. W.H. Freeman, San Francisco, California, $364 \mathrm{p}$.

[5]. Flury, J., Rummel, R., 2009. On the geoid - quasigeoid separation in mountain areas. Journal of Geodesy, 83: 829 - 847.

[6]. Sjöberg, Lars E., 2018. On the geoid and orthometric height vs. quasigeoid and normal height. Published Online: 2018-12-31 | DOI: https://doi.org/10.1515/jogs-2018-0011

[7]. Sjöberg, Lars E., 2010. A strict formula for geoid - to - quasigeoid separation. Journal of Geodesy, 84: 699 - 702, DOI 10.1007/s00190-010-0407-1

[8]. Tscherning, C., Forsberg, RE., Knudsen, P., 1992. The Gravsoft package for geoid determination. First Workshop on the geoid In Europe "Towards a Precise Pan-European Reference Geoid for the nineties". Prague, may 11-14.

(Xem tiếp trang 35) 